\title{
Identificación de malestares músculo-esqueletales en una planta de manufactura en Los Olivos, Lima-Perú
}

\author{
María Julia Brunette* \\ Universidad de Massachusetts, Lowell \\ César Morocho Albarracín* \\ Universidad de Massachusetts Lowell \& Producción y Planeamiento, Yobel SCM \\ María Teresa Noriega Araníbar* \\ Universidad de Lima, Perú \\ Nathalie Andrade* \\ Gerencia de Manufactura, Yobel SCM \\ Recibido: 25 de agosto del 2016 / Aprobado: 14 de setiembre del 2016
}

RESUMEN: El presente artículo describe el proceso de aplicación de una encuesta de evaluación músculo-esquelética en una planta de fabricación de joyas en Lima, la capital industrial del Perú. El cuestionario utilizado se diseñó siguiendo los principios de la ergonomía participativa, y respetando aspectos socioculturales de la población objetivo.

Palabras clave: salud ocupacional / ergonomía participativa / riesgo ergonómico / prevención / trastornos músculo-esqueléticos / calidad de vida / trabajadores / encuestas / metodología participativa

\section{Identifying Musculoskeletal Discomfort in a Manufacturing Plant in Los Olivos, Lima-Peru}

ABstRact: This article describes the process of implementing a musculoskeletal worker survey in a worker manufacturing plant in Lima, Peru's industrial capital. The questionnaire was designed following the principles of participatory ergonomics and respecting the various socio-cultural aspects of our target population.

Keywords: occupational health / participatory ergonomics / ergonomic risk / prevention / musculoskeletal disorders / quality of life / worker / survey / participatory methodology

* Correos electrónicos: maria_brunette@uml.edu, cesar_morochoalbarracin@ student.uml.edu, manorieg@ulima.edu.pe y nandrade@yobelscm.biz 


\section{INTRODUCCIÓN}

Marco teórico de la ergonomía participativa. El uso de la ergonomía participativa ha tenido resultados concretos en la mejora de las condiciones de trabajo, la productividad y la calidad de vida del trabajador (Haukka et al., 2008; Hess, 2004; Kogi, 2006; Laitinen et al., 1998; Liker et al., 1989; Loisel et al., 2001; Rivilis et al., 2008; Vink et al 1995), en la reducción de accidentes de trabajo (Lanoie y Tavenas, 1996) y en la mejora de procesos a nivel organizacional (Haims y Carayon, 1997; Maciel, 1998). El concepto de ergonomía participativa surgió hace aproximadamente tres décadas. Brown (1990) introdujo el concepto de hacer partícipes a los trabajadores dentro del estudio ergonómico de las estaciones de trabajo.

Básicamente, la ergonomía participativa promueve el bienestar físico, mental y social del trabajador a través de rediseños de tareas, estaciones de trabajo, herramientas, etc., en los que su opinión, es decir, la $v o z$ del trabajador, es respetada y valorada, pues es considerado un experto que conoce mejor que nadie los pequeños detalles y pormenores dentro del sistema de trabajo (Haines y Wilson, 1998; Kuorinka y Patry, 1995). Durante la década del noventa y a inicios del nuevo siglo, surgieron muchos estudios en los que se implementó y evaluó el impacto de realizar intervenciones para mejorar diversas condiciones de trabajo, no solo en el sector manufactura sino también en el área de servicios (Helali et al., 2008; Moore y Garg, 1996; Moore y Garg, 1998; Rosecrance y Cook, 2000; Vink et al., 1997).

En la figura 1 se puede apreciar la forma como la ergonomía participativa pretende atacar los factores de estrés físico y psicológico, con la inclusión de los trabajadores en la evaluación y diseño de las oportunidades de mejora, de tal modo que se evite el desarrollo de lesiones (Haines, 1998).

Antecedentes del proyecto. La empresa Yobel Supply Chain Management (Yobel SCM) desarrolla desde octubre del 2011 un proyecto denominado "Mejora de la Calidad de Vida del Trabajador de Yobel". Este proyecto tiene como objetivo general identificar las relaciones entre los elementos del sistema de trabajo y la calidad de vida del trabajador: estrés, satisfacción con el trabajo, estado de salud. Para ello, utiliza los principios que ofrece la ergonomía participativa, los cuales han sido bien documentados (Hignett et al., 2005; Kuorinka, 1997; Nagamachi, 1995). El objetivo fundamental del proyecto es mejorar las condiciones 
Identificación de malestares músculo-esqueletales en una planta de manufactura

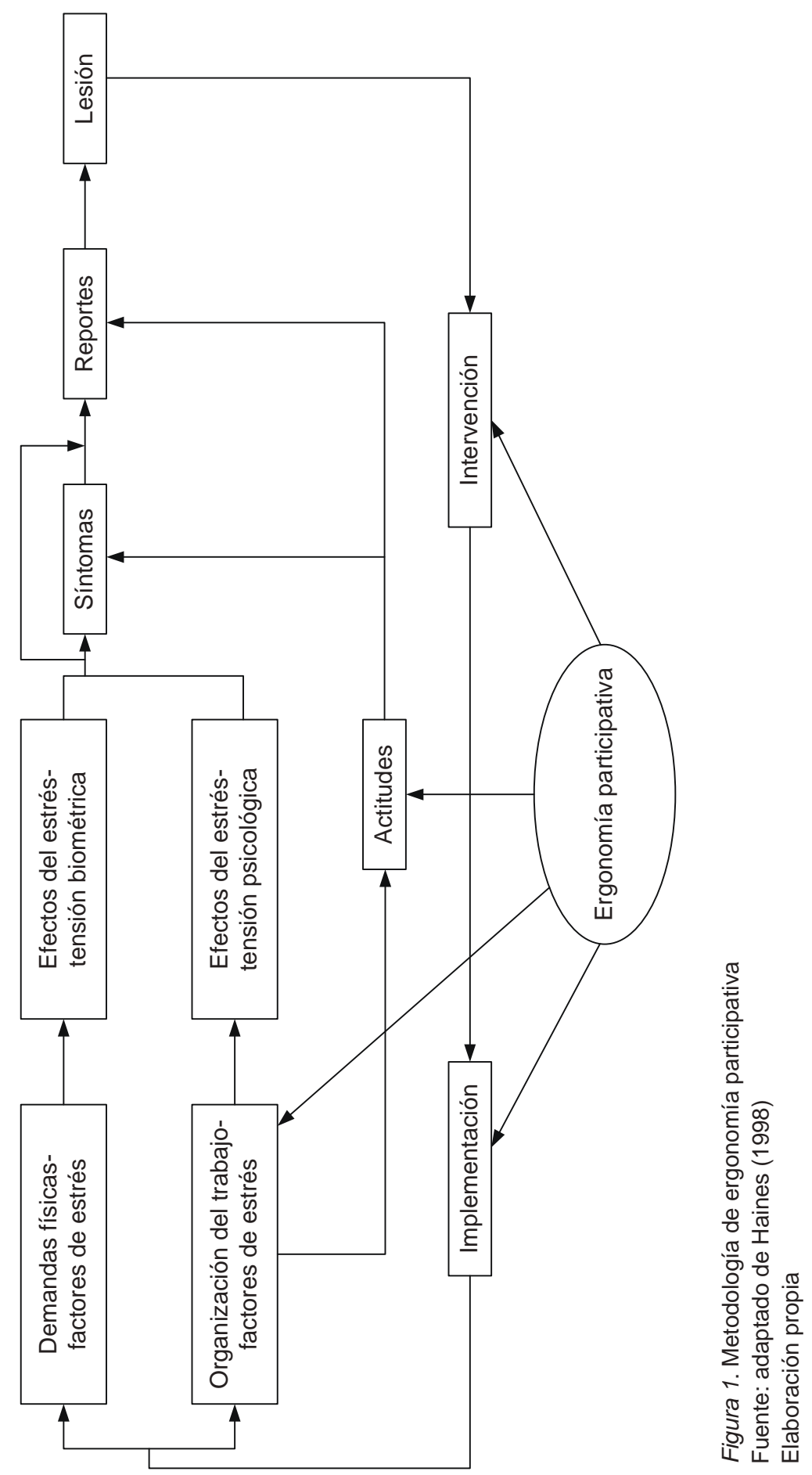


de trabajo, con el propósito de crear un impacto en la calidad de vida de los trabajadores. El presente estudio forma parte del estudio global denominado Calidad de Vida del Obrero Industrial Peruano.

La información presentada en este artículo se divide en dos fases: a) la identificación de riesgos ergonómicos; y b) la medición de la percepción del trabajador. Para conseguir este objetivo, primero se llevó a cabo un levantamiento de información del sistema de trabajo en las líneas de producción para identificar ciertos riesgos de salud. Luego, se realizó un análisis de la situación actual a partir de la aplicación de encuestas y la elaboración de informes de evaluación de riesgos ergonómicos en el ambiente de trabajo. En función del análisis en mención, la empresa, junto con el equipo de investigación, ha planteado oportunidades de mejora orientadas a perfeccionar las condiciones de trabajo a partir de los riesgos identificados. En todo momento se consultó al trabajador a través de entrevistas semiestructuradas, grupos focales y actividades grupales, en los que los trabajadores plasmaron su sentir y opinión sobre el impacto del trabajo en su salud y bienestar físico, mental y social empleando figuras del cuerpo humano.

Como parte del proyecto, en el año 2013 se realizó una encuesta en la planta de fabricación de joyas de la empresa Yobel SCM, que tenía como objetivo medir la percepción del trabajador respecto a molestias músculo-esqueletales y a la magnitud de las mismas. La encuesta forma parte de una visión innovadora de la empresa por empezar a incorporar consideraciones ergonómicas dentro de sus estaciones de trabajo. Este hecho impacta directamente en el bienestar de los trabajadores y en general de la propia empresa, aspecto comprobado en publicaciones que abordan la relación entre la productividad y la ergonomía (Gómez, 2002; Vink et al., 2006). Además, la ergonomía es considerada un factor de ventaja competitiva por parte de una empresa (Kuorinka y Patry, 1995; López, 2012; Maciel, 1998).

La encuesta forma parte de nuestra metodología participativa, en lo que se incluye al trabajador en la valoración de los riesgos a los que se ve expuesto (Manero, 2004). Se debe resaltar que las metodologías participativas permiten que las conclusiones acerca de los riesgos más significativos en el ambiente de trabajo sean más precisas (Kogi, 2006; Rubiol, 2012). 


\section{METODOLOGÍA}

\subsection{Diseño de encuesta para la toma de datos}

La encuesta aplicada es una adaptación de la versión presentada por Hagberg (1996). La encuesta original cuenta con dos partes: a) figura del cuerpo humano para sombrear donde se tenga alguna dolencia o malestar; b) grado de molestia.

Para su aplicación, se pide al trabajador que medite por un breve momento sobre cómo se siente previo a la realización de la encuesta. Luego, en función de esa meditación, se pide que proceda a realizar la encuesta. En la primera parte, se indica a los trabajadores que sombreen en dos siluetas del cuerpo humano (una con vista frontal y otra con vista posterior) aquellas zonas donde percibe malestar en ese momento. En la segunda parte, se solicita al trabajador que precise la magnitud del dolor que siente en la zona afectada. Para ello, se emplea una escala del 1 al 10, en la que 1 representa la ausencia de dolor y 10 una sensación intolerable de dolor.

Para la encuesta que se aplicó en Yobel SCM durante la segunda mitad del año 2013, se mantuvo la primera parte intacta y solo se realizaron modificaciones a la segunda parte. En esta última parte de la encuesta, en lugar de emplear una escala del 1 al 10, se optó por contar con una escala con tres niveles. Estos niveles se representaron a través de tres rostros, los cuales fueron: una cara feliz, una cara seria y una cara triste. La cara feliz indicaba que no sentían ningún dolor; la seria, que sentían un dolor leve; y la triste, que el dolor era muy fuerte. A partir de las reuniones con el equipo, se determinó el empleo de los rostros, con el objetivo de que la encuesta sea más amigable para los trabajadores. Además, con respecto a la escala, se redujo la cantidad de rubros de 10 a 3 , con el objetivo de que sea sencillo para el trabajador determinar la magnitud del dolor que podía sentir. Esta decisión se tomó teniendo en cuenta que los encuestados tienen un nivel educativo limitado.

Asimismo, en la encuesta se pide al trabajador que indique el área donde realizaba sus labores durante la realización de la encuesta. Debido a que en la planta de joyas se cuenta con una distribución por proceso, al identificar el área se tiene claridad de las operaciones que el trabajador estaba realizando. De esta manera, se puede asociar las dolencias que se han indicado en la encuesta con el área de trabajo, las posturas adoptadas, sus esfuerzos y frecuencia. La figura 2 muestra el diseño final de la encuesta aplicada para la planta de joyas. 


\section{Encuesta de evaluación músculo-esquelética}

Fecha: / /2013

Código:

Nombre:

Área:

Edad:

$18-44$ años
$45-59$ años
60 a más

Género: Masculino

Femenino

$($ )

$($ )

Piense acerca de cómo se siente en este momento:

1. Sombree el área en la figura donde tenga alguna dolencia o malestar. Puede sombrear varias áreas según su caso.
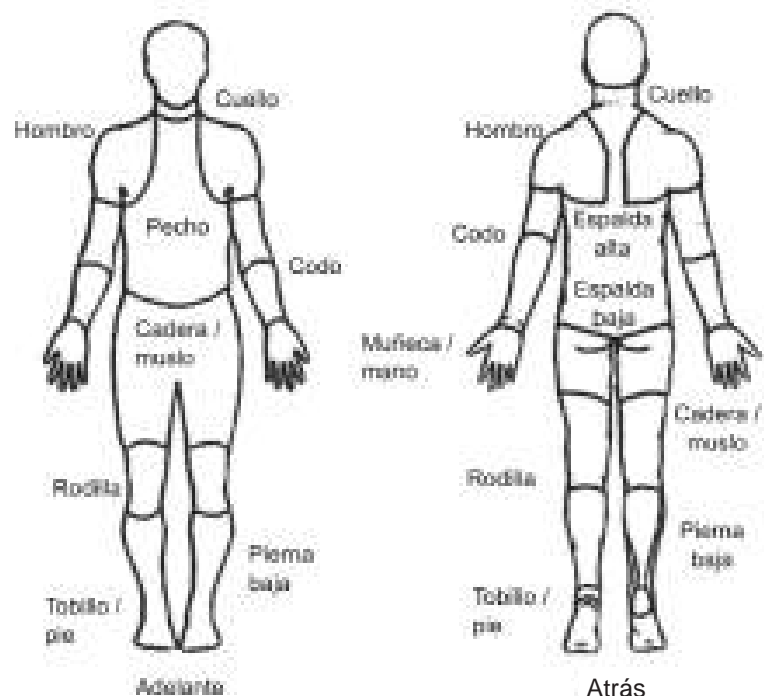

2. Usando la siguiente escala, por favor indique el grado de malestia que siente en ambos lados del cuerpo
No duele ; ;
Duele poco $:-$
Duele mucho :

\begin{tabular}{|c|c|c|c|c|c|c|c|c|c|c|c|c|}
\hline \multirow[b]{3}{*}{ Hombro } & \multicolumn{6}{|c|}{ Adelante } & \multicolumn{6}{|c|}{ Atrás } \\
\hline & \multicolumn{3}{|c|}{ Lado derecho } & \multicolumn{3}{|c|}{ Lado izquierdo } & \multicolumn{3}{|c|}{ Lado derecho } & \multicolumn{3}{|c|}{ Lado izquierdo } \\
\hline & ;) & ; & : & ;) & 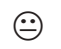 & $\theta$ & ;: & $\odot$ & : & ;: & ;) & : \\
\hline Pecho & ;) & $\odot$ & : & ;: & :) & : & ;) & $\odot$ & $:$ & ;:) & :) & : \\
\hline Codo/ antebrazo & ;) & $\odot$ & : & ;: & $\odot$ & : & ;) & :) & : & ;) & 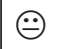 & : \\
\hline Mano/ muñeca & ;: & $\odot$ & : & ;: & 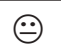 & : & ;) & ; & : & ;) & ;) & : \\
\hline Cadera/ muslo & ;) & $\odot$ & : & ;) & :- & $\theta$ & ;) & 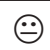 & : & ;:) & :) & : \\
\hline Cuello & ;: & $\odot$ & $:$ & ;) & $:-$ & : & ;) & 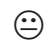 & : & ;: & ; & : \\
\hline Pierna baja & ;) & $\odot$ & : & ;: & 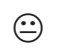 & (2) & ;) & $\odot$ & : & ;:) & $\odot$ & : \\
\hline Tobillo/ pie & ;) & ; & : & ;: & $\odot$ & : & ;) & $\odot$ & $:$ & ;) & $\odot$ & 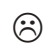 \\
\hline Otros: & ;) & $\odot$ & : & ;: & 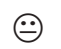 & : & ;) & $\odot$ & : & ;) & $\odot$ & (2) \\
\hline
\end{tabular}

Figura 2. Encuesta malestares/síntomas músculo-esqueléticos Elaboración propia 


\subsection{Muestra}

En las actividades por área de trabajo (según se muestra en la tabla 1) se realizan operaciones que suponen sobreesfuerzos, posturas forzadas y/o movimientos repetitivos. La conjunción de estos factores de riesgo pueden generar alteraciones músculo-esqueléticas (Bravo, 1988). Por ello, realizar una encuesta de riesgos músculo-esqueléticos permite tener una noción acerca de las partes del cuerpo que se ven más afectadas en la actividad laboral. Con ello, resulta más eficaz la implementación de medidas correctivas.

Para la determinación de la muestra requerida, se empleó la fórmula que se muestra a continuación:

$$
\mathrm{n}=\frac{\mathrm{N} \times \mathrm{Z}^{2} \times \mathrm{p} \times(1-\mathrm{p})}{(\mathrm{N}-1) \times \mathrm{e}^{2}+\mathrm{Z}^{2} \times \mathrm{p} \times(1-\mathrm{p})}
$$

Se debe tener en cuenta que $\mathrm{n}$ representa el tamaño de muestra, $\mathrm{N}$ es el tamaño de la población, $\mathrm{Z}$ es el nivel de confianza, e es el margen de error y p es la probabilidad de éxito. Las consideraciones que se tuvieron en cuenta tienen un nivel de confianza de $99 \%(\mathrm{Z}=2,575)$, un margen de error de $5 \%$, una probabilidad de éxito de 0,5 y la cantidad de trabajadores de la planta de joyas es 236 .

Por ello, luego de reemplazar los valores en la formula indicada, se obtiene que la muestra debe ser de 175 trabajadores para conseguir un nivel de confianza de $99 \%$ y un margen de error de $5 \%$.

\section{Tabla 1}

Descripción de actividades por área

\begin{tabular}{lll}
\hline \multicolumn{1}{c}{ Área } & \multicolumn{1}{c}{ Descripción de actividades } \\
\hline $1 . \quad$ Acabado & $\begin{array}{l}\text { Los colaboradores realizan operaciones de engomado } \\
\text { y pegado. Para el engomado, colocan goma en las } \\
\text { cavidades de la joya con la ayuda de una jeringa. Para el } \\
\text { pegado, colocan piedras pequeñas sobre las cavidades } \\
\text { de la joya, en donde previamente se ha colocado goma. }\end{array}$ \\
\hline $2 . \quad$ Almacén & $\begin{array}{l}\text { Los colaboradores entregan y almacenan los insumos } \\
\text { para el proceso productivo. Para ello, trasladan cajas } \\
\text { de diferentes pesos (peso promedio: } 8 \mathrm{~kg} \text { ). Además, } \\
\text { emplean una computadora para registrar los insumos que } \\
\text { ingresan y salen del almacén. }\end{array}$ \\
\end{tabular}


(continuación)

\begin{tabular}{lll}
\hline \multicolumn{1}{c}{ Área } & \multicolumn{1}{c}{ Descripción de actividades } \\
\hline 3. Aseguramiento & Los colaboradores realizan controles de calidad en \\
de la calidad & $\begin{array}{l}\text { las diferentes áreas del proceso productivo. Además, } \\
\text { ejecutan auditorías esporádicas al proceso. Por ello, se } \\
\text { encuentran en constante movimiento durante su jornada } \\
\text { de trabajo. }\end{array}$ \\
\hline 4. $\quad$ Centrifugado & $\begin{array}{l}\text { Los colaboradores cuentan con dos puestos de trabajo } \\
\text { diferenciados: el maquinista y el operario de línea. El } \\
\text { maquinista se encarga de la inyección de metal fundido } \\
\text { sobre el molde. Se trabaja de pie y en constante } \\
\text { movimiento de brazos. Este trabajo es realizado } \\
\text { únicamente por varones. El operario de línea se encarga } \\
\text { de retirar las piezas del molde; para ello, emplea pinzas } \\
\text { con las cuales ejerce fuerza. }\end{array}$ \\
\hline
\end{tabular}

5. Cera perdida Los colaboradores cuentan con los puestos de trabajo de inyección, yeso, casting, rebaje y selección. En el caso de la inyección, se realizan las siguientes operaciones: inyección de cera en moldes con la ayuda de una máquina, retoque de los anillos de cera formados con la ayuda de un bisturí, armado de un árbol de anillos de cera con la ayuda de un piro grabador. En el caso del yeso, se forma un molde de yeso con la ayuda de una máquina y el árbol de cera. En el caso de casting, se emplea el molde de yeso para formar un árbol íntegramente de metal, a partir del cual se pueden obtener las joyas al cortar los extremos de las hojas del árbol.

\begin{tabular}{lll}
\hline 6. & Colgado & $\begin{array}{l}\text { Los colaboradores arman un bastidor con las joyas } \\
\text { correspondientes. Para ello, elaboran tiras de alambres, } \\
\text { en las cuales amarran las joyas. }\end{array}$ \\
\hline 7. & Descolgado & Los colaboradores retiran las joyas de los bastidores. \\
\hline $8 . \quad$ Encajado & $\begin{array}{l}\text { Los colaboradores colocan los productos terminados } \\
\text { dentro de cajas. }\end{array}$ \\
\hline $9 . \quad$ Epóxico & $\begin{array}{l}\text { Los colaboradores elaboran diferentes combinaciones de } \\
\text { pinturas. Luego, con la ayuda de una jeringa y un pedal, } \\
\text { se coloca la cantidad requerida de pintura en la joya } \\
\text { correspondiente. }\end{array}$ \\
\hline 10. & Galvánica & $\begin{array}{l}\text { Los colaboradores sumergen los bastidores con joyas en } \\
\text { las diferentes tinas que hay en el área. De esta manera, } \\
\text { se realiza el bañado de las joyas, el cual puede ser en } \\
\text { plata o en oro. }\end{array}$ \\
\hline $11 . \quad$ Joyería & $\begin{array}{l}\text { Los colaboradores elaboran la primera joya patrón en } \\
\text { función del diseño enviado por el área de modelado. }\end{array}$ \\
\hline
\end{tabular}

(continúa) 
(continuación)

\begin{tabular}{|c|c|c|}
\hline & Área & Descripción de actividades \\
\hline 12. & Linkera & $\begin{array}{l}\text { Los colaboradores se encargan de realizar la unión de } \\
\text { dos joyas. Para ello, emplean una máquina que realiza la } \\
\text { unión con el accionar de un pedal. }\end{array}$ \\
\hline 13. & Modelado & $\begin{array}{l}\text { Los colaboradores diseñan en computadora una joya. } \\
\text { Para ello, se encuentran la mayor parte del tiempo } \\
\text { sentados y emplean mucho el mouse. }\end{array}$ \\
\hline 14. & Moldes & $\begin{array}{l}\text { Los colaboradores diseñan el molde que se empleará } \\
\text { para replicar la joya elaborada por el área de joyería. Para } \\
\text { ello, usan un bisturí, el cual permite trazar los canales por } \\
\text { donde pasará el metal para la formación de la joya. }\end{array}$ \\
\hline 15. & $\begin{array}{l}\text { Programa de } \\
\text { reinserción laboral }\end{array}$ & $\begin{array}{l}\text { Los colaboradores rotan en actividades que no suponen } \\
\text { riesgos ergonómicos elevados. Este programa está } \\
\text { dirigido a las personas que cuentan con una enfermedad } \\
\text { ocupacional. Se debe resaltar que la selección de las } \\
\text { actividades se realiza en colaboración con el área médica } \\
\text { de la empresa. }\end{array}$ \\
\hline 16. & Prototipo & $\begin{array}{l}\text { Los colaboradores se encargan de apoyar al área de } \\
\text { joyería en la realización del patrón joyero. Para ello, } \\
\text { trasladan el patrón por las áreas necesarias para que } \\
\text { pueda ser elaborado. }\end{array}$ \\
\hline 17. & Rebaje & $\begin{array}{l}\text { Los colaboradores realizan un pulido de las joyas con } \\
\text { el objetivo de que no existan imperfecciones. Para ello, } \\
\text { constantemente se encuentran frotando las joyas sobre } \\
\text { los esmeriles. }\end{array}$ \\
\hline 18. & Retoques & $\begin{array}{l}\text { Los colaboradores realizan el acondicionamiento de todas } \\
\text { las piezas patrones. Para ello, desempeñan operaciones } \\
\text { de lijado y pulido con la ayuda de un esmeril. }\end{array}$ \\
\hline 19. & Soldado & $\begin{array}{l}\text { Los colaboradores realizan la operación de soldadura de } \\
\text { las joyas correspondientes. }\end{array}$ \\
\hline 20. & Vibrado & $\begin{array}{l}\text { Los colaboradores colocan las joyas al interior de las } \\
\text { máquinas correspondientes, las cuales se encargan de } \\
\text { que la joya cuente con una superficie lisa. }\end{array}$ \\
\hline 21. & Limpieza & $\begin{array}{l}\text { Los colaboradores se encargan de la limpieza en toda la } \\
\text { planta de joyas (parte administrativa y de producción). }\end{array}$ \\
\hline
\end{tabular}

Elaboración propia 


\subsection{Aplicación de la encuesta}

Previo a la encuesta, se realizó un estudio piloto con algunos trabajadores de la planta. El objetivo de esta fase fue evaluar la facilidad en el llenado de la encuesta y, como parte de una metodología participativa, contemplar la opinión del personal. Además, se capacitó a todo el personal de la planta en el llenado de la encuesta y se les explicó el objetivo de la misma. Esto se logró a través de una charla de cinco minutos en los días previos al inicio de la encuesta.

La encuesta se aplicó del 17 de septiembre al 2 de octubre del 2013. Se precisó que la encuesta debía realizarse al finalizar la jornada, para que los colaboradores indiquen los malestares que sentían al momento de resolver la encuesta. En ese sentido, la encuesta refleja el malestar percibido producto de la jornada de trabajo. Como se indicó en la tabla 1, la encuesta se aplicó a todas las áreas de producción de la planta de joyas, la cuales incluyen 21 áreas: acabados, almacén, aseguramiento de la calidad, centrifugado, cera perdida, colgado, descolgado, encajado, epóxico, galvánica, joyería, linkera, modelado, moldes, prototipo, rebaje, retoques, soldado, vibrado, limpieza y descolgado. Además, se incluyó al programa de reinserción laboral.

\section{RESULTADOS}

La tabla 2 muestra la cantidad de encuestas en cada una de las áreas de la sección joyas. Adicionalmente, se puede apreciar el porcentaje encuestado para cada una de las áreas. En casi todas se encuestó a más del $50 \%$ de los empleados, en algunos casos al $100 \%$, con un saldo final de $78 \%$. Se debe resaltar que, con el objetivo de no afectar el proceso productivo de la planta, la encuesta se realizó en reemplazo del tiempo que se destina diariamente a las charlas de seguridad, las cuales suponen cinco minutos por día. Como se mencionó anteriormente, se pretendía que la encuesta se aplique al finalizar la jornada de trabajo. Sin embargo, esto no se pudo cumplir en la totalidad de las áreas, debido a que el horario de las charlas de seguridad varía según las necesidades del proceso de producción. Por ello, en las áreas de centrifugado, moldes, retoques, colgado y descolgado se realizó a mitad de la jornada de trabajo. 
Tabla 2

Cantidad de personas por área $\left(^{*}\right)$

\begin{tabular}{|c|c|c|c|}
\hline Área & $\begin{array}{l}\text { Promedio de } \\
\text { encuestados }\end{array}$ & $\begin{array}{l}\text { Cantidad } \\
\text { de personas } \\
\text { por área }\end{array}$ & $\begin{array}{c}\text { Porcentaje } \\
\text { encuestado } \\
\text { por área }\end{array}$ \\
\hline Cera perdida & 5 & 5 & $100 \%$ \\
\hline Colgado & 8 & 8 & $100 \%$ \\
\hline Linkera & 2 & 2 & $100 \%$ \\
\hline Modelado & 2 & 2 & $100 \%$ \\
\hline Retoques & 8 & 8 & $100 \%$ \\
\hline Soldado & 2 & 2 & $100 \%$ \\
\hline Centrifugado & 39 & 40 & $98 \%$ \\
\hline Aseguramiento de la calidad & 5 & 6 & $83 \%$ \\
\hline Joyería & 5 & 6 & $83 \%$ \\
\hline Rebaje & 5 & 6 & $83 \%$ \\
\hline Almacén & 8 & 10 & $80 \%$ \\
\hline Moldes & 14 & 18 & $78 \%$ \\
\hline Acabados & 23 & 30 & $77 \%$ \\
\hline Vibrado & 6 & 8 & $75 \%$ \\
\hline Galvánica & 11 & 15 & $73 \%$ \\
\hline Epóxico & 10 & 15 & $67 \%$ \\
\hline Encajado & 19 & 30 & $63 \%$ \\
\hline Prototipo & 3 & 5 & $60 \%$ \\
\hline $\begin{array}{l}\text { Programa de reinserción } \\
\text { laboral }\end{array}$ & 4 & 8 & $50 \%$ \\
\hline Limpieza & 1 & 2 & $50 \%$ \\
\hline Descolgado & 4 & 10 & $40 \%$ \\
\hline Total & 184 & 236 & $78 \%$ \\
\hline
\end{tabular}

Nota: $\left(^{*}\right)$ En la tabla 1 se aprecia la descripción de las operaciones realizadas en cada área Elaboración propia 
Durante los 11 días de la toma de datos, la encuesta se aplicó en promedio a 184 personas por día. La tabla 3 muestra la cantidad de personas que completaron la encuesta a diario. La cantidad de personas por día varía durante el periodo de toma de datos, puesto que hubo días en que algunas áreas, por un tema productivo, no pudieron destinarle tiempo a la realización de la encuesta. Sin embargo, como se aplicó durante 11 días, al juntar los datos recogidos en las diferentes jornadas, la disparidad no afectó significativamente las conclusiones que se obtuvieron de la data recopilada. Además, un estudio acerca de la continuidad de malestares músculo-esqueléticos en trabajadores de la industria de plásticos concluyó que los malestares en mención se perciben durante más de una semana. Asimismo, se debe recalcar que la cantidad de encuestados en promedio es superior al tamaño de la muestra necesario para poder predecir los resultados con un nivel de confianza de $99 \%$ y un margen de error de $5 \%$. Así, confirmamos que el análisis refleja los malestares percibidos por el colaborador.

Tabla 3

Cantidad de personas por fecha

\begin{tabular}{ll}
\hline Fecha & $\mathbf{n}$ \\
\hline $17 / 09 / 2013$ & 168 \\
$19 / 09 / 2013$ & 188 \\
$20 / 09 / 2013$ & 131 \\
$23 / 09 / 2013$ & 189 \\
$24 / 09 / 2013$ & 207 \\
$25 / 09 / 2013$ & 190 \\
$26 / 09 / 2013$ & 202 \\
$27 / 09 / 2013$ & 178 \\
$30 / 09 / 2013$ & 148 \\
$01 / 10 / 2013$ & 176 \\
$02 / 10 / 2013$ & 173 \\
Total & 1950 \\
\hline
\end{tabular}

Elaboración propia 


\subsection{Identificación de la molestia músculo-esquelética}

Como se mencionó inicialmente, la primera parte de la encuesta pretendía identificar aquellas áreas del cuerpo en las que se percibían malestares. En ese sentido, a continuación se muestra un gráfico con los porcentajes promedio de incidencia en cada parte del cuerpo durante los 11 días de duración de la encuesta. Se debe mencionar que estos porcentajes promedio se obtuvieron a partir de un promedio simple de los porcentajes que corresponden a las partes del cuerpo en cada una de las áreas que participaron en la encuesta.

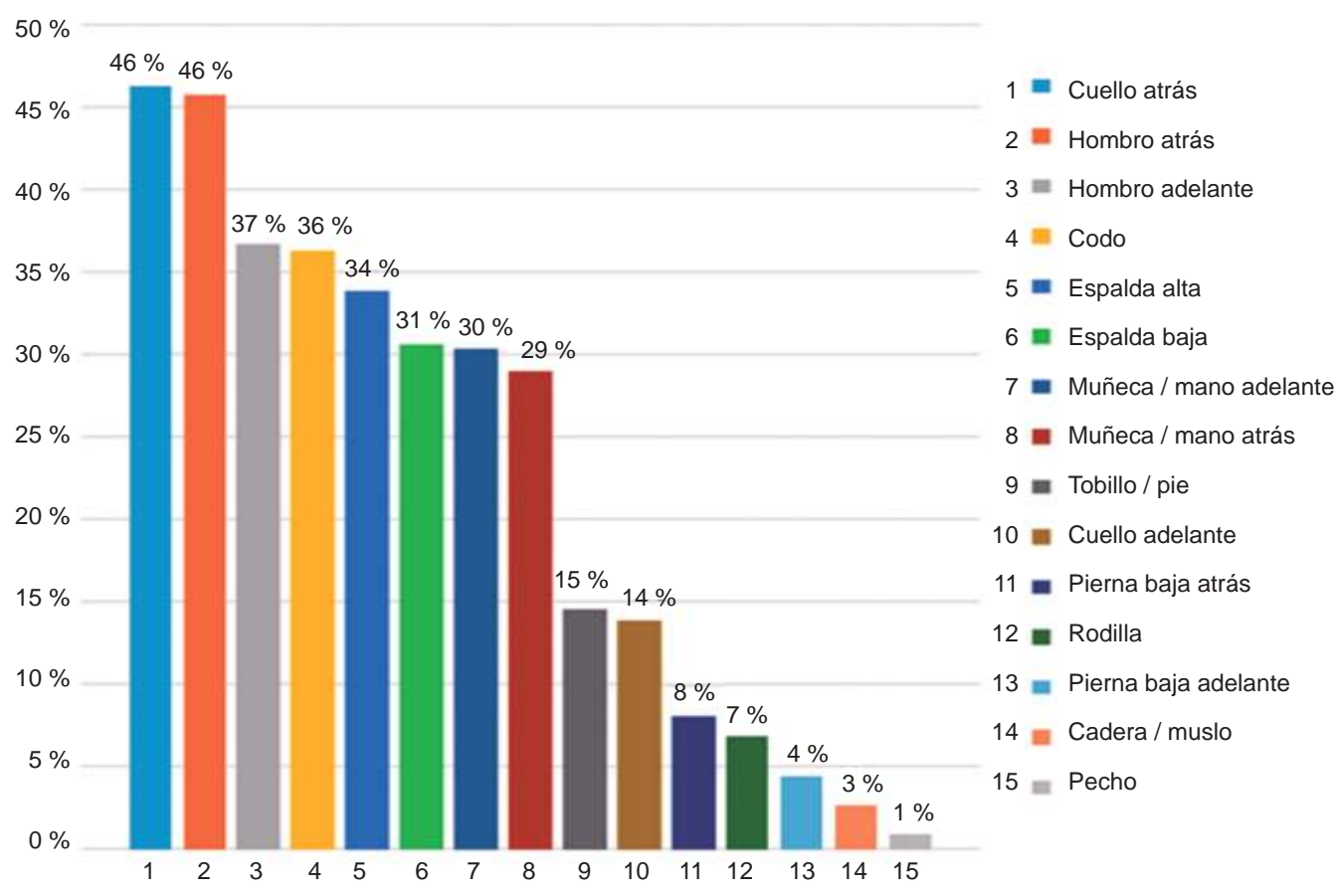

Figura 3. Síntomas músculo-esqueléticos para todas las áreas (11 días) Elaboración propia

Como se puede apreciar en la figura, las principales partes del cuerpo en donde se sombreó una dolencia en la primera pregunta fueron el cuello y los hombros. El $46 \%$ de los encuestados indicaron que sentían malestar en estas dos zonas, el cual puede asociarse a posturas de trabajo inadecuadas que se presentan en diferentes áreas de la planta. Resulta importante recalcar que las posturas de trabajo 
forzadas suponen un gran riesgo de generar lesiones músculo-esqueléticas (Vernaza y Sierra, 2005). Incluso, algunos investigadores sostienen que dentro de la industria manufacturera, uno de los principales factores de riesgo de lesiones músculo-esqueléticas son las posturas incorrectas (Fernández, 2007). Este hecho también ha sido afirmado en un estudio realizado para valorar la carga postural en trabajadores del rubro de metalmecánica (Montiel, 2006). Asimismo, se puede resaltar que la principal zona afectada es el cuello, una zona con dolores recurrentes en diversas industrias, entre ellas la metalmecánica (Hernandez, 2012).

Por su lado, la tercera parte de encuestados, aproximadamente un $30 \%$, indicó malestares en codo, espalda, muñecas y manos. Un hecho que llamó particularmente la atención fue que la principal parte del cuerpo en donde se manifestaron malestares no hayan sido las manos ni las muñecas, debido a que, por el tipo de trabajo que se realiza en las diversas áreas de la planta, hay un constante movimiento repetitivo de muñecas. Sin embargo, a nivel general, para los colaboradores este hecho no es tan significativo en comparación con el dolor que perciben en otras partes del cuerpo como hombros, cuello y codos. Un hecho similar se evidenció en un estudio de evaluación de desórdenes músculo-esqueléticos, en el que se concluyó que hay mayor percepción de dolor en los miembros superiores del cuerpo en comparación con otras partes del mismo (Reyes, 2012).

En los siguientes gráficos se muestra la forma como se distribuyeron los porcentajes para las áreas que agrupan a la mayor cantidad de trabajadores. También se resalta aquellas áreas consideradas críticas por los riesgos ergonómicos que suponen las operaciones. En el área de centrifugado, más del $45 \%$ de encuestas indicaron malestar en hombros. Asimismo, una de cada tres encuestas (aproximadamente un $33 \%$ ) indicó molestias en muñecas, manos, cuello y espalda.

En el área de acabados, un poco más del 60 \% de encuestados indicó malestar en cuello y hombros. Aproximadamente un $40 \%$ indicó molestias en codos y espalda, mientras que alrededor del $30 \%$ manifestó dolencias en muñecas y manos. 


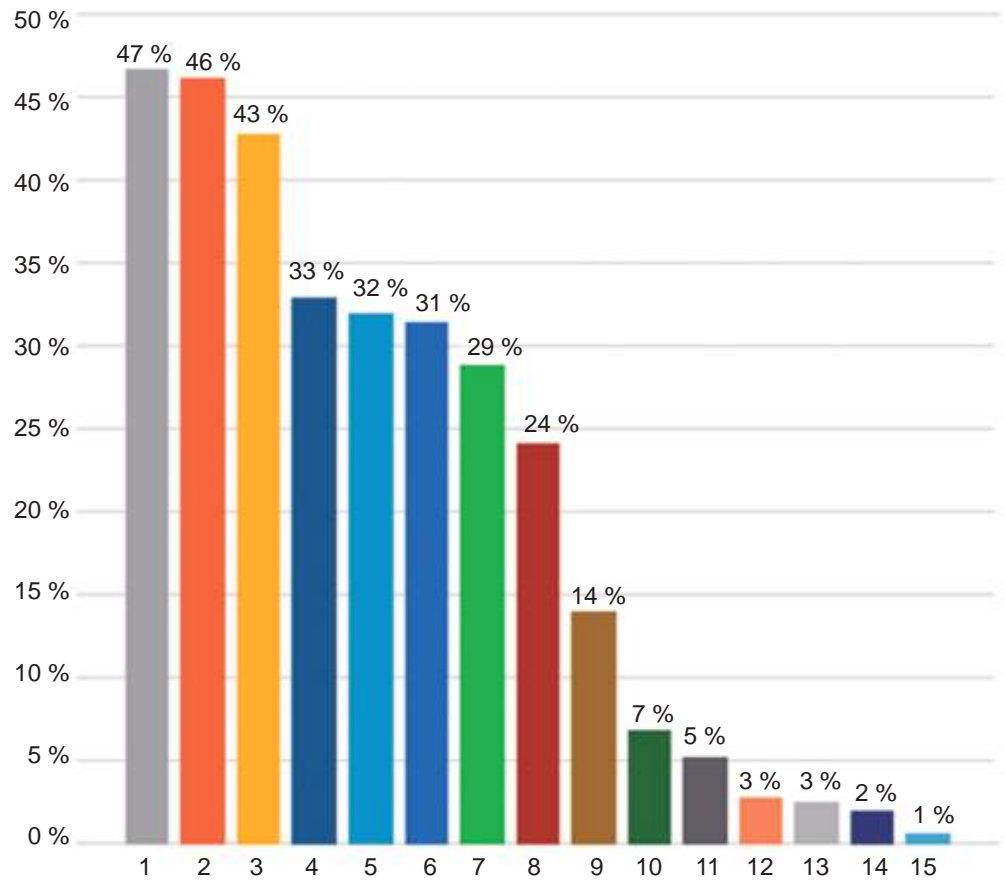

$1=$ Hombro adelante

2 프 Hombro atrás

$3=$ Codo

4 ㅂ.ㄹñeca / mano adelante

5 = Cuello atrás

6 Espalda alta

7 Espalda baja

8 auñeca / mano atrás

9 = Cuello adelante

10 므 Rodilla

11 a Tobillo / pie

12 = Cadera / muslo

$13=$ Pecho

14 ․ Pierna baja atrás

15 = Pierna baja adelante

Figura 4. Síntomas músculo-esqueléticos para el área de centrifugado (11 días)

Elaboración propia

$70 \%$

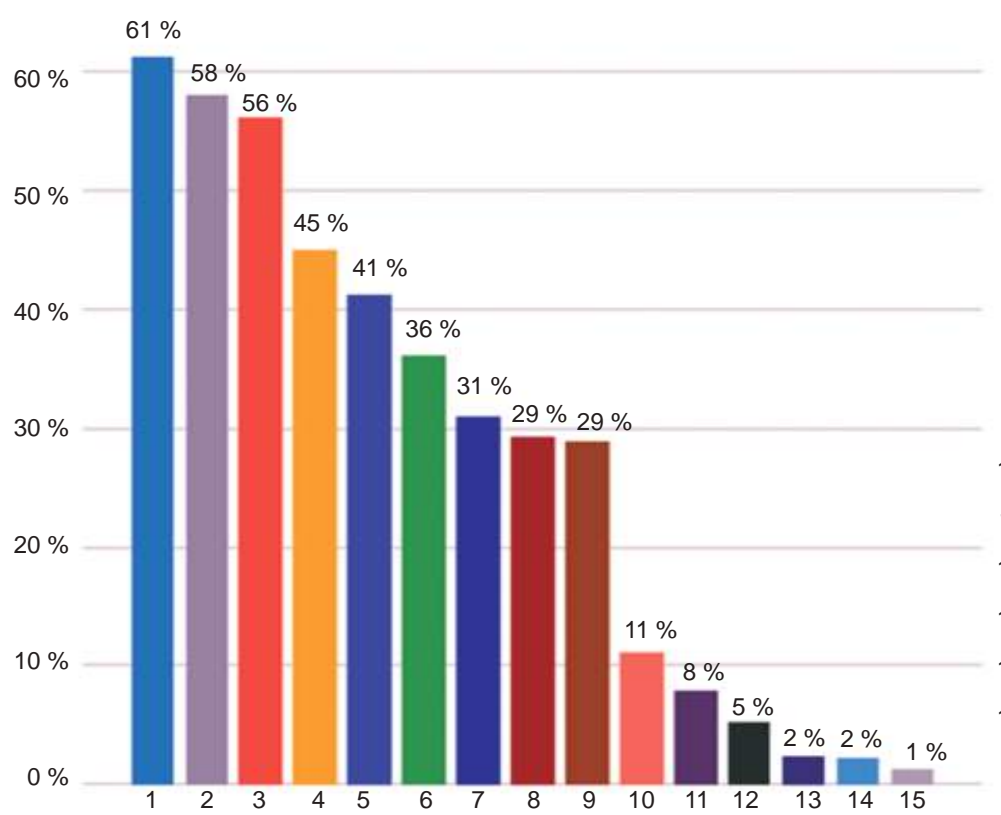

1 E Cuello atrás

2 플 Hombro adelante

$3=$ Hombro atrás

4 프 Codo

5 토 Espalda alta

6 Espalda baja

7 - Muñeca / mano adelante

8 ․ Muñeca / mano atrás

9 = Cuello adelante

$10=$ Cadera / muslo

11 = Tobillo / pie

12 - Rodilla

13 - Pierna baja atrás

14 - Pierna baja adelante

$15=$ Pecho

Figura 5. Síntomas músculo-esqueléticos para el área de acabados (11 días)

Elaboración propia 


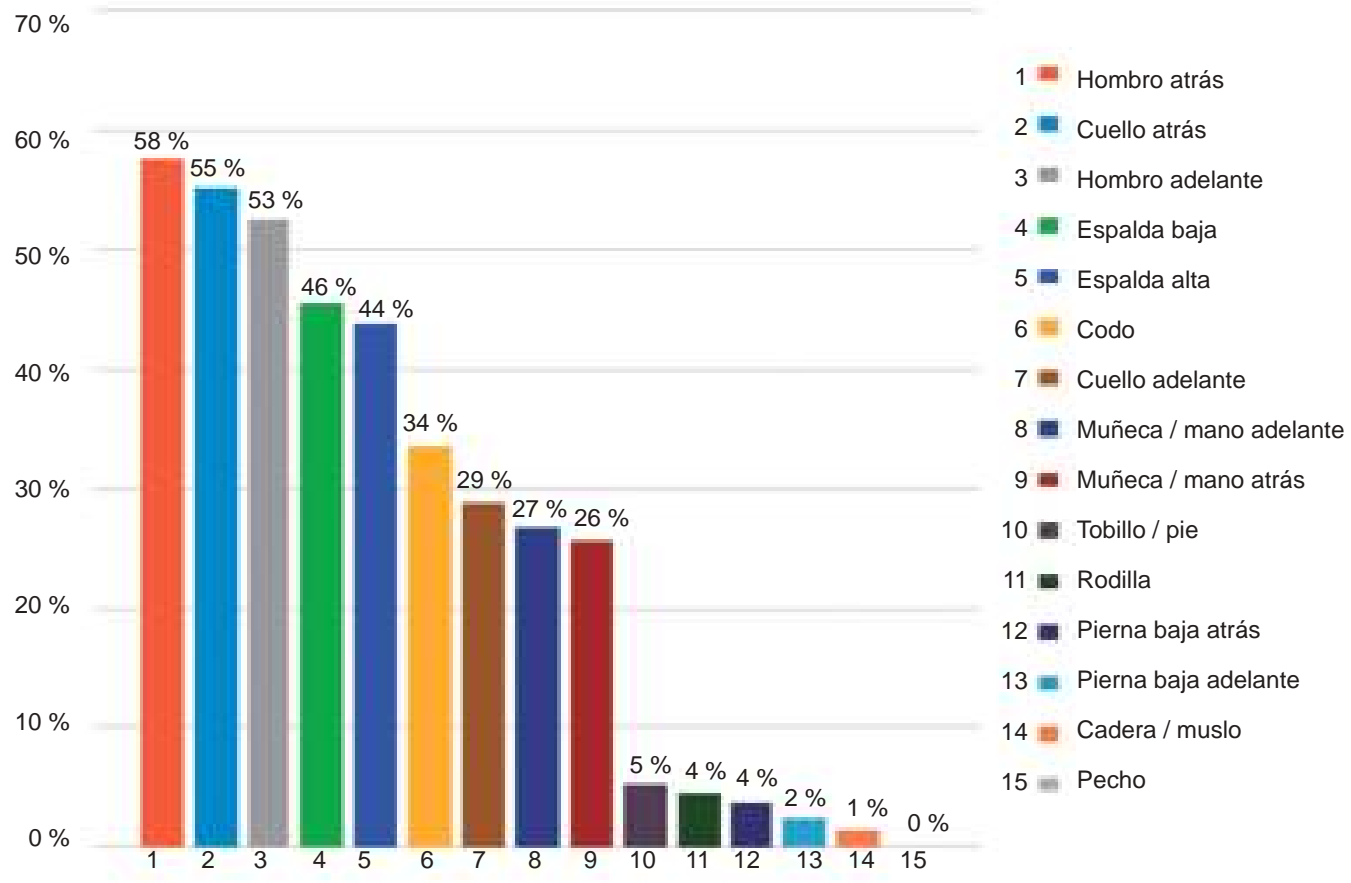

Figura 6. Síntomas músculo-esqueléticos para el área de encajado (11 días) Elaboración propia

$70 \%$

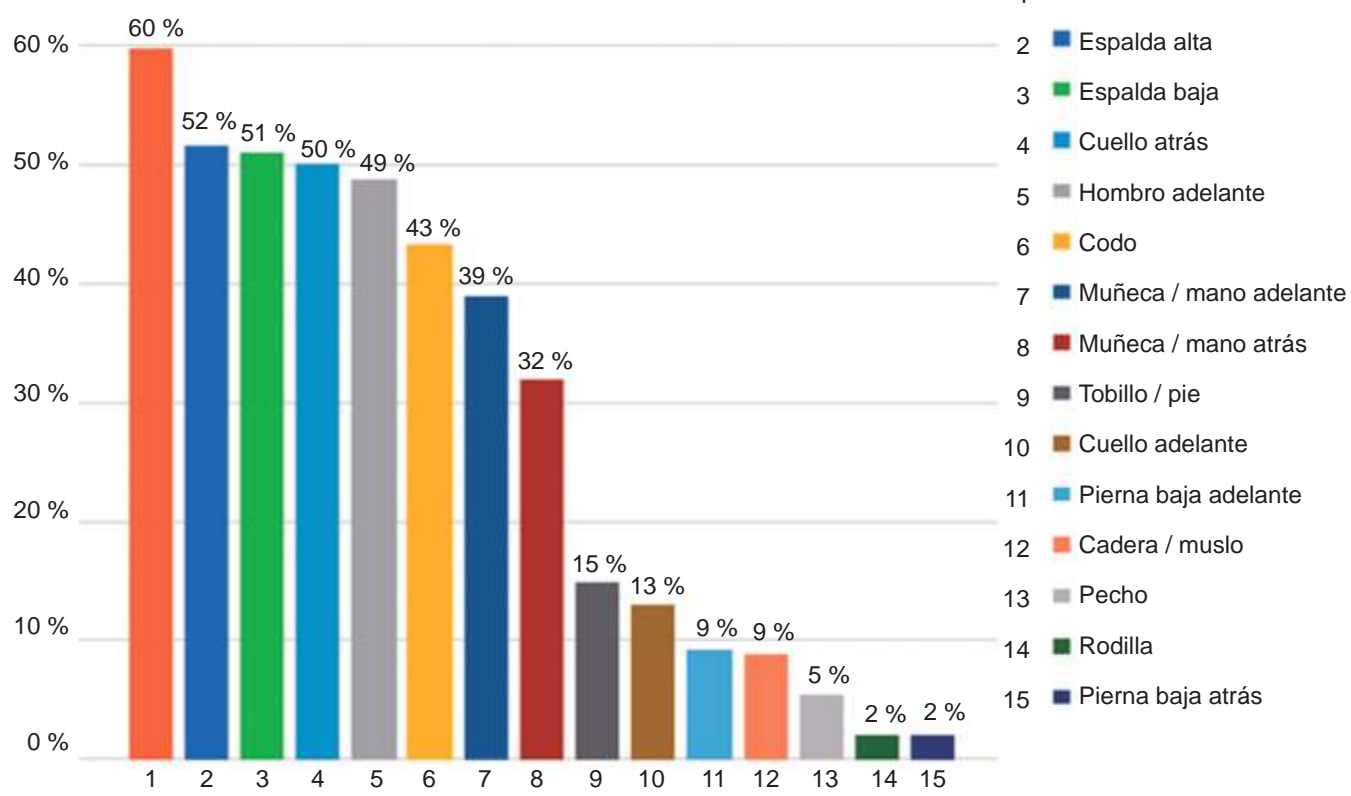

Figura 7. Síntomas músculo-esqueléticos para el área de colgado (11 días) Elaboración propia 
En el área de encajado, la principal parte del cuerpo resaltada fue el hombro posterior, con aproximadamente un $60 \%$. A su vez, en más del $50 \%$ de encuestas se indicó malestar en cuello, hombro adelante y espalda baja.

En el área de colgado, más del 50 \% de encuestados indicaron malestar en hombros, espalda y cuello. Asimismo, resulta importante destacar que aproximadamente en el $40 \%$ de las encuestas se observó malestar en codo, muñeca y manos.

En el área de galvánica, más del 60 \% de colaboradores revelaron malestares en hombro, cuello, tobillo y pie. Además, un número superior al $40 \%$ del personal indicó molestias en la espalda y pierna baja.

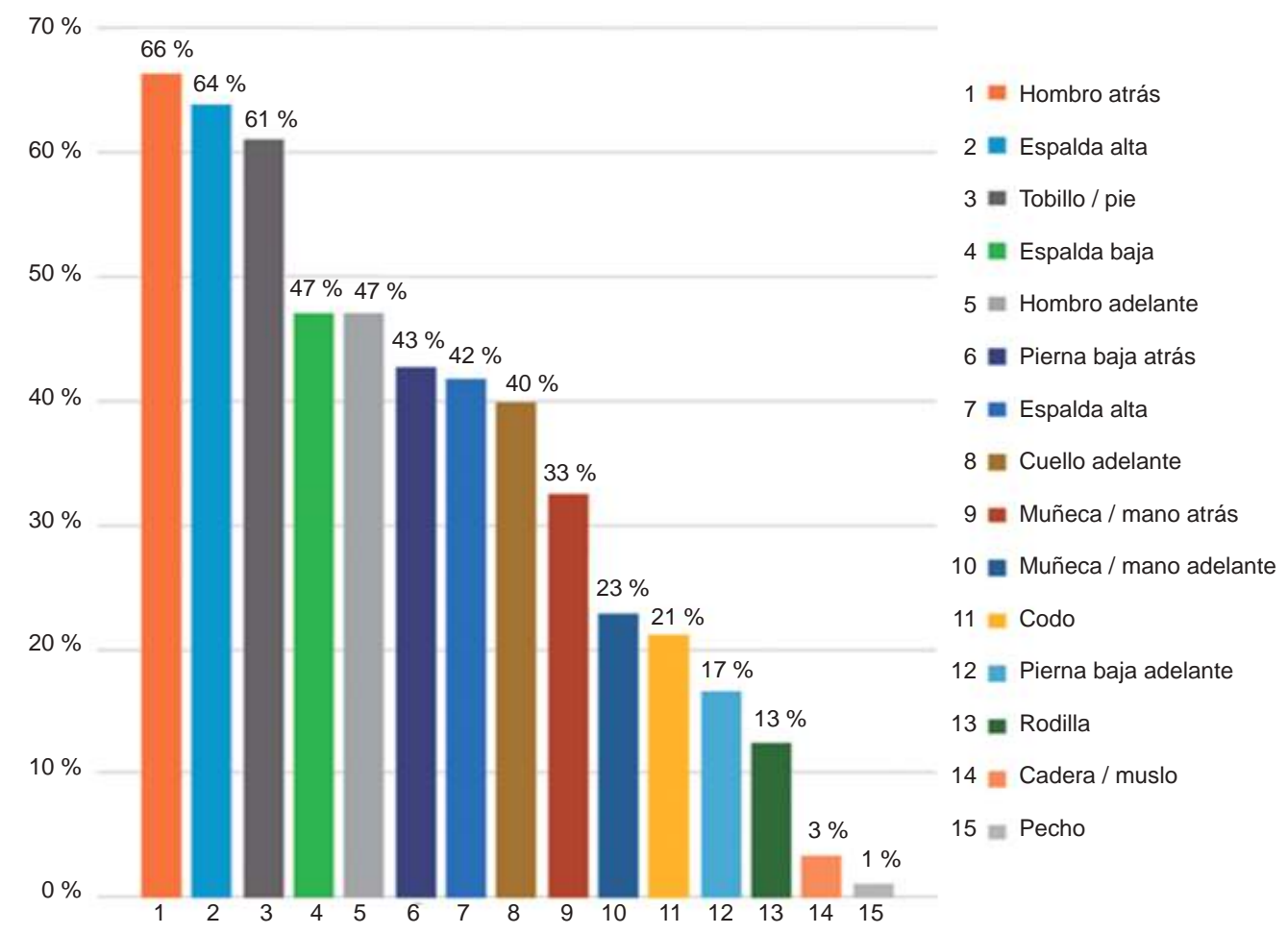

Figura 8. Síntomas músculo-esqueléticos para el área de galvánica (11 días) Elaboración propia 
Porcentaje promedio de caras (área: todas)

\begin{tabular}{|c|c|c|c|}
\hline & \multicolumn{3}{|c|}{ Cara } \\
\hline & Feliz & Seria & Triste \\
\hline Hombro adelante derecha & $60 \%$ & $25 \%$ & $15 \%$ \\
\hline Hombro adelante izquierda & $71 \%$ & $21 \%$ & $8 \%$ \\
\hline Hombro atrás derecha & $57 \%$ & $27 \%$ & $16 \%$ \\
\hline Hombro atrás izquierda & $64 \%$ & $25 \%$ & $11 \%$ \\
\hline Pecho derecha & $96 \%$ & $4 \%$ & $1 \%$ \\
\hline Pecho izquierda & $95 \%$ & $4 \%$ & $1 \%$ \\
\hline Espalda alta derecha & $79 \%$ & $15 \%$ & $6 \%$ \\
\hline Espalda alta izquierda & $81 \%$ & $13 \%$ & $7 \%$ \\
\hline Codo / antebrazo adelante derecha & $68 \%$ & $24 \%$ & $8 \%$ \\
\hline Codo / antebrazo adelante izquierda & $77 \%$ & $18 \%$ & $5 \%$ \\
\hline Codo / antebrazo atrás derecha & $72 \%$ & $21 \%$ & $8 \%$ \\
\hline Codo / antebrazo atrás izquierda & $80 \%$ & $15 \%$ & $6 \%$ \\
\hline Mano / muñeca adelante derecha & $59 \%$ & $30 \%$ & $11 \%$ \\
\hline Mano / muñeca adelante izquierda & $71 \%$ & $23 \%$ & $6 \%$ \\
\hline Mano / muñeca atrás derecha & $66 \%$ & $26 \%$ & $9 \%$ \\
\hline Mano / muñeca atrás izquierda & $73 \%$ & $20 \%$ & $6 \%$ \\
\hline Cadera / muslo adelante derecha & $92 \%$ & $6 \%$ & $2 \%$ \\
\hline Cadera / muslo adelante izquierda & $93 \%$ & $5 \%$ & $2 \%$ \\
\hline Espalda baja derecha & $79 \%$ & $15 \%$ & $7 \%$ \\
\hline Espalda baja izquierda & $81 \%$ & $13 \%$ & $6 \%$ \\
\hline Cuello adelante derecha & $80 \%$ & $12 \%$ & $8 \%$ \\
\hline Cuello adelante izquierda & $84 \%$ & $11 \%$ & $6 \%$ \\
\hline Cuello atrás derecha & $68 \%$ & $21 \%$ & $12 \%$ \\
\hline Cuello atrás izquierda & $69 \%$ & $20 \%$ & $11 \%$ \\
\hline Pierna baja adelante derecha & $94 \%$ & $5 \%$ & $1 \%$ \\
\hline Pierna baja adelante izquierda & $94 \%$ & $5 \%$ & $1 \%$ \\
\hline Pierna baja atrás derecha & $92 \%$ & $6 \%$ & $2 \%$ \\
\hline Pierna baja atrás izquierda & $92 \%$ & $7 \%$ & $1 \%$ \\
\hline Tobillo / pie adelante derecha & $89 \%$ & $8 \%$ & $3 \%$ \\
\hline Tobillo / pie adelante izquierda & $93 \%$ & $5 \%$ & $2 \%$ \\
\hline Tobillo / pie atrás derecha & $84 \%$ & $12 \%$ & $4 \%$ \\
\hline Tobillo / pie atrás izquierda & $87 \%$ & $10 \%$ & $3 \%$ \\
\hline Rodilla derecha & $97 \%$ & $2 \%$ & $1 \%$ \\
\hline Rodilla izquierda & $99 \%$ & $1 \%$ & $0 \%$ \\
\hline
\end{tabular}

Elaboración propia 


\subsection{Magnitud de la percepción de la molestia músculo-esquelética}

En esta última parte de la encuesta, se pretende identificar qué tan significativa es la molestia manifestada por el colaborador. Para ello, mostramos un cuadro comparativo con los porcentajes promedio de incidencia de cara feliz, seria y triste para cada una de las partes del cuerpo. La tabla 4 indica el porcentaje promedio de colaboradores que marcaron cada una de las caras durante los 11 días que duró la encuesta. La tabla se encuentra ordenada en función de la parte del cuerpo que tuvo un porcentaje mayor de caras serias y tristes en conjunto, lo cual indica presencia de síntomas/malestares músculo-esqueletales. Además, se puede apreciar que dentro de las 10 partes del cuerpo en donde se manifestó un mayor porcentaje de caras serias y tristes se encuentran: hombros, manos, muñecas, cuello, codos y antebrazos.

De este conjunto de partes del cuerpo, es importante resaltar los hombros y el cuello, debido a que fueron las zonas más señaladas en la primera parte de la encuesta. Por ello, se concluye que, a nivel de toda la planta de joyas, en promedio la mayor parte de colaboradores percibe un malestar en hombros y cuello. Asimismo, en estas partes del cuerpo la sensación de dolor es más significativa en comparación con otras zonas. Cabe resaltar que hubo coincidencia entre las partes del cuerpo que se indicaron en la primera y segunda parte de la encuesta. Este hecho permite validar la veracidad de los resultados obtenidos.

\section{DISCUSIÓN}

El estudio pretende encontrar la raíz que causa los malestares de los trabajadores industriales, con el objetivo de plantear e implementar rediseños ergonómicos en las estaciones de trabajo. Para este fin, se han contemplado estudios previos que buscan analizar las estaciones de trabajo en base a la aplicación de cuestionarios como parte de la ergonomía aplicada (Cruz y Garnica, 2010). Por ejemplo, destacamos un estudio realizado en el Perú para conocer la percepción de los trabajadores de manufactura sobre su estación de trabajo a partir de la aplicación de una encuesta (Brunette, 2011). De otro lado, se puede mencionar un estudio realizado en Brasil sobre la identificación de malestares músculo-esqueléticos en trabajadores de la industria plástica a través del análisis de una encuesta (Fernandes, 2011). Resultaría sumamente beneficioso para el estudio poder contrastar los resultados 
de las percepciones de los trabajadores con evaluaciones médicas. Por esa razón, hemos relacionado nuestras conclusiones con un estudio realizado en Colombia que incluyó evaluaciones médicas en el análisis de riesgo de lesiones músculo-esqueléticas en trabajadores dedicados a la floricultura (Barrero, 2012).

A partir de la aplicación de la encuesta de evaluación músculoesquelética, se determinó que las zonas de cuello y hombros son las que suponen un mayor malestar por parte de los trabajadores. Este hecho resulta de suma importancia, ya que, debido al tipo de actividades realizadas -como centrifugado, descolgado, rebaje y retoques-en la planta de joyas, el malestar en cuello y hombros se puede asociar a posturas forzadas que adoptan los trabajadores en sus estaciones de trabajo. No obstante, se debe resaltar que las zonas de manos y muñecas, las cuales están más asociadas a movimientos repetitivos, tuvieron una relevancia menor para los trabajadores.

En ese sentido, diversos estudios hacen referencia a la criticidad de factores de riesgo a nivel ergonómico, como las posturas forzadas, sobreesfuerzo y movimientos repetitivos (Hernández, 2012). Sin embargo, en este caso se desprende que los colaboradores perciben con mayor intensidad en su jornada de trabajo malestares producto de posturas forzadas en comparación con los ocasionados por movimientos repetitivos.

\section{Impacto del trabajo bajo posturas inadecuadas: percepción de los trabajadores}

Como se mencionó anteriormente, al momento del llenado de la encuesta se pidió a los trabajadores que coloquen los malestares que percibían en el momento en que se entregaba la encuesta, el cual fue en su mayoría al finalizar la jornada de trabajo. De esa manera, los malestares percibidos están relacionados a la labor del día. Por ello, se entiende que, producto de la jornada de trabajo, los colaboradores perciben con mayor intensidad la postura forzada en comparación al movimiento repetitivo. Se debe resaltar que ambos constituyen factores de riesgo a nivel ergonómico y, según comenta Vernaza (2005), tienen una asociación directa con dolores músculo-esqueléticos. Asimismo, a partir de la encuesta, los trabajadores manifestaron una percepción que se inclinaba por un mayor malestar en las posturas forzadas. Para comprender este hecho, según Estrada (2011), debemos considerar que el 
desarrollo de traumas acumulativos producto de movimientos repetitivos se generan a largo plazo, mientras que los malestares ocasionados por problemas de posturas forzadas se perciben a corto plazo, por ejemplo, al finalizar cada jornada, como manifestaron los trabajadores en la encuesta.

\section{Rediseño de estaciones de trabajo involucrando la opinión del trabajador}

Siguiendo las pautas generales y los principios de acción de la ergonomía participativa repasados en la sección inicial, cabe mencionar que el proyecto global Calidad de Vida se ha constituido en un estudio pionero dentro del área de la ergonomía participativa en el Perú. Como muestra clara de cómo la investigación-acción puede lograr cambios concretos, se pide al lector revisar los materiales audiovisuales desarrollados directamente con los trabajadores de Yobel SCM, donde se siguió un proceso iterativo, incorporando la reacción/opinión del trabajador en las decisiones finales (Brunette, 2014).

Por otro lado, la identificación de las zonas en las cuales los trabajadores sentían malestares producto de la realización de sus labores permitió identificar oportunidades de mejora en el diseño de sus estaciones de trabajo. Específicamente, cuando se determinó que el cuello y los hombros eran las zonas en las que los trabajadores percibían mayor malestar, se buscó rediseñar la estación de trabajo, para que el trabajador adopte una postura que evite la inclinación excesiva del cuello, así como el levantamiento de los brazos por encima del nivel del hombro. Como parte del proceso de rediseño de las estaciones de trabajo, se empezó con una prueba piloto del cambio de la estación de trabajo. Esto con el propósito de realizar una simulación de la mejora y obtener la retroalimentación de los trabajadores sobre el cambio. Por ello, se fabricó una mesa adicional para el área, la cual consideró los cambios que buscaban ofrecer una mejor postura de trabajo. Por ejemplo, en la figura 9 se aprecia la mesa piloto que se implementó en el área de acabados, la cual posee reposacodos adheridos a la mesa con regulación de inclinación y un soporte regulable en inclinación sobre la superficie de la mesa.

A partir de la prueba piloto, los trabajadores presentaron sugerencias sobre la propuesta de rediseño. Por ejemplo, en función de sus comentarios, se redujeron las dimensiones de los reposacodos para 
María Brunette, César Morocho, María Teresa Noriega, Nathalie Andrade
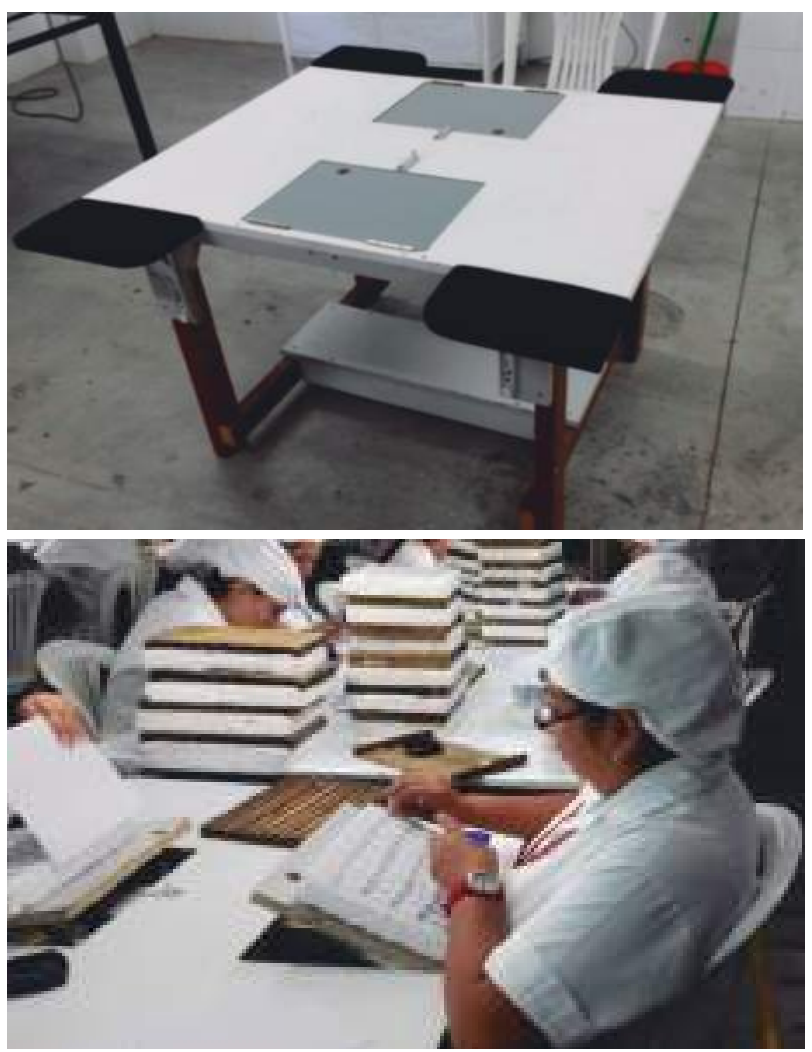

Figura 9. Piloto de rediseño de estación de trabajo Elaboración propia

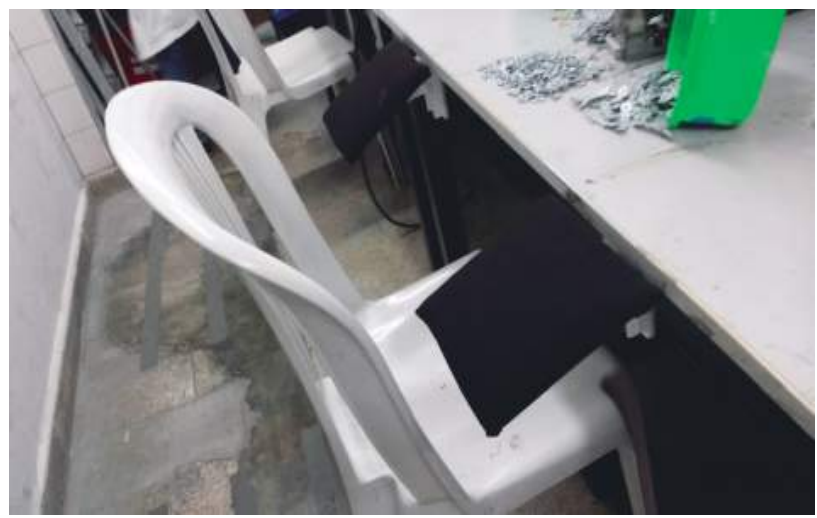

Figura 10. Rediseño de estación de trabajo Elaboración propia 
ofrecer mayor comodidad al trabajador. En la figura 10 se muestra el diseño final de los reposacodos.

Luego de la retroalimentación de los trabajadores, se pudo definir un diseño final para la estación de trabajo, el cual se implementará de manera progresiva en cada estación del área. Se debe resaltar el aporte de los trabajadores a la validación del rediseño de la estación de trabajo. En ese sentido, según comenta Brunette (2014), es importante contar con la contribución directa de los trabajadores de la empresa, con el objetivo de plantear medidas eficaces para mejorar las condiciones laborales.

\section{Limitaciones del estudio}

Se debe tener en cuenta que la planta de joyas de la empresa Yobel SCM tiene una situación particular. Esto se debe a que la mayoría de trabajadores son de género femenino. Por ese hecho, la proporción de mujeres en la planta es aproximadamente un $90 \%$. Y, en consecuencia, los resultados obtenidos tienen una marcada tendencia de género respecto de la percepción de malestares a partir de las actividades realizadas en la planta de joyas. Además, se debe tener presente que las conclusiones del estudio se basan en las percepciones de los trabajadores respecto de los malestares generados a partir de su labor diaria. Los mismos no han sido contrastados con un diagnóstico médico que permita validar los malestares identificados.

\section{Recomendaciones de política}

Sería de suma importancia que la ley de seguridad y salud en el trabajo (Ley N. 29783) y la norma básica de ergonomía (RM N. 375-2008-TR) contemplen metodologías participativas como parte de las propuestas de mejora de las condiciones de trabajo. Esto debido a que son los trabajadores quienes, al estar en contacto directo con las operaciones, tienen mayor claridad sobre las oportunidades de mejora que permitirán agilizar su labor. Asimismo, en su jornada diaria perciben de manera directa las consecuencias de una adecuada o deficiente estación de trabajo. Por ello, son los más interesados en que la situación de la misma mejore. Se debe resaltar que hay antecedentes de estudios previos, por ejemplo, Brunette (2003), en el que se hace mención de la necesidad de 
contrastar nuestra normativa respecto de lo establecido por la Organización Internacional del Trabajo, de tal modo que tengamos un marco legal laboral que posibilite una adecuada calidad de vida.

\section{CONCLUSIONES}

- El empleo de la encuesta de evaluación músculo-esquelética posibilita la identificación de malestares músculo-esqueletales en la persona. Por ejemplo, la aplicación de la encuesta permitió determinar que el malestar más agudo percibido por los trabajadores se ubica en el cuello y los hombros. Por ello, el diseño y la implementación de una encuesta de evaluación músculo-esquelética constituye un instrumento importante en el análisis de riesgos ergonómicos en empresas industriales.

- Tras analizar los resultados de la encuesta, se pudo hacer una jerarquización de los riesgos ergonómicos presentes en la planta de joyas a partir de la percepción de dolor manifestada por los trabajadores. Por ejemplo, los trabajadores percibían con mayor intensidad el impacto de trabajar bajo posturas forzadas en comparación con los movimientos repetitivos.

- Los resultados del análisis ergonómico permitieron orientar las oportunidades de mejora hacia el factor de riesgo de posturas forzadas en las estaciones de trabajo de la planta de joyas. Por ello, se realizó un planteamiento de rediseño de estaciones de trabajo en el que se adoptan posturas óptimas, mejorando la percepción de los colaboradores y contribuyendo a reducir la incidencia de lesiones músculo-esqueléticas.

- Utilizar una metodología participativa en los estudios ergonómicos permite direccionar de manera eficaz el planteamiento de oportunidades de mejora en las estaciones de trabajo. En este estudio, la metodología fue aplicada en la identificación de riesgos a través del diseño e implementación de la encuesta de evaluación músculo-esquelética y en el rediseño de las estaciones de trabajo a partir de la aplicación de pruebas piloto, que permitieron contar con sugerencias de los trabajadores previo al cambio de la estación de trabajo. De esta manera, se pretende lograr mejoras y cambios sostenibles en la salud, la productividad y el bienestar general. 


\section{REFERENCIAS}

Barrero, L., et al. (2012). Physical workloads of the upper-extremity among workers of the Colombian flower industry. Am. J. Ind. Med., 55, 926-939.

Bravo, P y Chicharro, E. (1988). Problemas posturales músculo esquelético en el trabajo. La salud en el trabajo, 3, 249-264.

Brown, O. (1990). Marketing participatory ergonomics: current trends and methods to enhance organizational effectiveness. Ergonomics, 33, 601-604.

Brunette, M. J. (2003). Satisfacción, salud y seguridad ocupacional en el Perú. Consorcio de investigación económica y social. Economía y Sociedad, 49, 47-52.

Brunette, M. J., Smith, M. J., y Punnett, L. (2011). Perceptions of Working and Living Conditions among Industrial Male and Female Workers in Perú. WORK, A Journal of Prevention, Assessment \& Rehabilitation, 38, 211-223.

Brunette, M. J. and Morocho, C. (2014). Musculoskeletal discomfort at work? Let's ask the people. GOHNET Newsletter, 24, 9-10.

Brunette, M. (2014). Calidad de Vida del Obrero Industrial Peruano (Quality of Working Life among Industrial Workers in Perú). 3 partes [videograbación] Intimedia. University Umass Lowell y Yobel SCM.

Brunette, M. (2014). Elisa: I work for my family. [videograbación]. Recuperado de http://www.uml.edu/Research/WHOCC/videos. aspx. Lima, Perú.

Brunette, M. (2014). Maribel: Everything is possible in life. [videograbación]. Recuperado de http://www.uml.edu/Research/WHOCC videos.aspx. Lima, Perú.

Brunette, M. (2014). Pedro: You cannot work without good health. [videograbación]. Recuperado de http://www.uml.edu/Research/ WHOCC/videos.aspx. Lima, Perú.

Cruz, G. y Garnica, G. (2010). Ergonomía aplicada. Bogotá: Ecoe Ediciones.

Estrada, M. (2011). Ergonomía. Medellín: Editorial - Universidad de Antioquia. 
Fernandes, R. (2007). Los trastornos músculo-esqueléticos: un reto para los prevencionistas. Gestión de práctica de riesgos laborales, 42, $12-22$.

Fernandes, R., et al. (2011). Prevalence of musculoskeletal disorders among plastics industry workers. Cadernos de Saúde Pública, 27, 78-86.

Gómez, C. et al. (2002). Ergonomía, historia y ámbitos de aplicación. Fisioterapia-Universidad de Murcia, 3-10.

Hagbergs, M. (1995). Work-related Musculoskeletal Disorders (WMSDs): A Reference Book for Prevention. London: Taylor \& Francis.

Haims, M. y Carayon, P. (1997). Theory and practice for the implementation of 'in-house', continuous improvement participatory ergonomic programs. Applied Ergonomics, 29, 461-472.

Haines, H. y Wilson, J. (1998). Development of a framework for participatory ergonomics. Institute for Occupational Ergonomics for the Health and Safety Executive.

Haukka, E. et al. (2008). A randomised controlled trial on whether a participatory ergonomics intervention could prevent musculoskeletal disorders. Occupational and Environmental Medicine, 65, 849-856.

Helali, F. et al (2008). Participatory ergonomics intervention in an industrially developing country - a case study. International Journal of Occupational Safety and Ergonomics, 14, 159-176.

Hernandez, J. et al. (2012). Factores de fatiga en operadores de maquinaria semiautomatizada en México. Ingeniería Industrial, 30, 11-27.

Hess, J. et al. (2004). A participatory ergonomics intervention to reduce risk factors for low-back disorders in concrete laborers. Applied ergonomics, 35, 427-441.

Hignett, S. et al. (2005). Finding ergonomic solutions - participatory approaches. Occupational Medicine, 55, 200-207.

Kogi, K. (2006). Participatory methods effective for ergonomic workplace improvement. Applied ergonomics, 37, 547-554. 
Kuorinka, I. (1997). Tools and means of implementing participatory ergonomics. International Journal of Industrial ergonomics, 19, 267-270.

Kuorinka, I. y Patry, L. (1995). Participation as a means of promoting occupational health. International Journal of Industrial Ergonomics, 15, 365-370.

Laitinen, H. et al. (1998). Improving physical and psychosocial working conditions through a participatory ergonomic process. A beforeafter study at an engineering workshop. International Journal of Industrial Ergonomics, 21, 35-45.

Lanoie, P. y Tavenas, S. (1996). Costs and benefits of preventing workplace accidents: the case of participatory ergonomics. Safety Science, 24, 181-196.

Liker, J. et al. (1989). A comparative analysis of participatory ergonomics programs in U.S. and Japan manufacturing plants. International Journal of Industrial Ergonomics, 3, 185-199.

López, V. et al. (2012). Ergonomía y productividad: variables que se relacionan con la competitividad de las plantas maquiladoras. Ingeniería Industrial, 17-32.

Maciel, R. (1998). Participatory ergonomics and organizational change. International Journal of Industrial Ergonomics, 22, 319-325.

Manero, A. et al. (2004). Un modelo simple para la evaluación integral del riesgo a lesiones músculo-esqueléticas (Modsi). Mapfre medicina, 16, 86-94.

Montiel, M. et al. (2006). Valoración de la carga postural y riesgo músculo esquelético en trabajadores de una empresa metalmecánica. Salud de los trabajadores, 14, 61-69.

Moore, J. y Garg, A. (1996). Use of participatory ergonomics teams to address musculoskeletal hazards in the red meat packing industry. American Journal of Industrial Medicine, 29, 402-408.

Nagamachi, M. (1995). Requisites and practices of participatory ergonomics. International Journal of Industrial ergonomics, 15, 371-377.

Reyes, J, et al. (2012). Evaluación de la limitación funcional causada por desórdenes músculo esqueléticos en miembros superiores, 
empleando el cuestionario QuickDash. Revista Colombiana de Medicina Física y Rehabilitación, 22, 11-18.

Rivilis, I. et al. (2008). Effectiveness of participatory ergonomic interventions on health outcomes: A systematic review. Applied Ergonomics, 39, 342-358.

Rosecrance, J. y Cook, T. (2000). The use of participatory action research and ergonomics in the prevention of work - related musculoskeletal disorders in the newspaper industry. Applied Occupational and Environmental Hygiene, 15, 255-262.

Vernaza, P. y Sierra, C. (2005). Dolor músculo-esquelético y su asociación con factores de riesgo ergonómicos en trabajadores administrativos. Revista Salud Pública, 7, 317-326.

Vink, P. et al. (1995). A participatory ergonomics approach to reduce mental and physical workload. International Journal of Industrial Ergonomics, 15, 389-396.

Vink, P. et al. (1997). A participatory ergonomics approach to redesign work of scaffolders. Safety science, 26, 75-85.

Vink, P. et al. (2006). Positive outcomes of participatory ergonomics in terms of greater comfort and higher productivity. Applied Ergonomics, 37, 537-546. 